Nonlinear Processes in Geophysics (2005) 12: 149-156

SRef-ID: $1607-7946 / \mathrm{npg} / 2005-12-149$

European Geosciences Union

(c) 2005 Author(s). This work is licensed

under a Creative Commons License.

\title{
Developing a dynamically based assimilation method for targeted and standard observations
}

\author{
F. Uboldi ${ }^{1}$, A. Trevisan ${ }^{2}$, and A. Carrassi ${ }^{3}$ \\ ${ }^{1}$ LEGOS/SHOM, Toulouse, France \\ ${ }^{2}$ CNR-ISAC, Bologna, Italy \\ ${ }^{3}$ Dipartimento di Fisica - Università di Ferrara, Italy
}

Received: 31 August 2004 - Revised: 22 December 2004 - Accepted: 7 January 2005 - Published: 28 January 2005

Part of Special Issue "Quantifying predictability"

\begin{abstract}
In a recent study, a new method for assimilating observations has been proposed and applied to a small size nonlinear model. The assimilation is obtained by confining the analysis increment in the unstable subspace of the Observation-Analysis-Forecast (OAF) cycle system, in order to systematically eliminate the dynamically unstable components, present in the forecast error, which are responsible for error growth. Based on the same ideas, applications to more complex models and different, standard and adaptive, observation networks are in progress. Observing System Simulation Experiments (OSSE), performed with an atmospheric quasi-geostrophic model, with a restricted "land" area where vertical profiles are systematically observed, and a wider "ocean" area where a single supplementary observation is taken at each analysis time, are reviewed. The adaptive observation is assimilated either with the proposed method or, for comparison, with a 3-D VAR scheme. The performance of the dynamic assimilation is very good: a reduction of the error of almost an order of magnitude is obtained in the data void region. The same method is applied to a primitive equation ocean model, where "satellite altimetry" observations are assimilated. In this standard observational configuration, preliminary results show a less spectacular but significant improvement obtained by the introduction of the dynamical assimilation.
\end{abstract}

\section{Introduction}

Data assimilation in meteorology and oceanography deals with high-dimensional chaotic systems. In such systems, errors which are present in the initial condition grow in time, due to instability. Other errors are of course introduced along

Correspondence to: F. Uboldi

(uboldi@magritte.it) the Observation-Analysis-Forecast (OAF) cycle. Model errors of various origin are introduced along the forecasting integration, and observation errors (including errors in the observation operator) are introduced at analysis time. Anyhow all these errors eventually increase or decrease depending on the action of the dynamical instabilities of the system. A relevant point, then, is observability (Ghil, 1997): how many perfect observations, under perfect model conditions, are necessary to exactly determine the state of the system? The answer depends on the number of independent unstable directions.

The OAF cycle is seen as a dynamical system, subject to observational forcing. Its perturbations (tangent linear) dynamics is, for analysis states:

$\delta \mathbf{x}_{k+1}^{a}=(\mathbf{I}-\mathbf{K H}) \mathbf{M} \delta \mathbf{x}_{k}^{a}$,

where $\mathbf{K}$ is the assimilation matrix (Kalman gain), $\mathbf{H}$ is the linearized observation operator, $\mathbf{M}$ is the tangent linear model and $\mathbf{I}$ is the identity matrix. It is the stability of such a system that characterizes the tendency of the sequence of analysis states (or the sequence of forecast states at assimilation times) to approach or depart from the trajectory of the real system, at least when the two trajectories are sufficiently close to each other. As a consequence, what is of concern is the dimension of the unstable subspace of such a forced system.

Generally, but depending on the specific implementation choices of each assimilation scheme, the forcing due to the assimilation of observations results in a reduction of the dimension of the unstable subspace. As a consequence, the order of the problem is reduced, and only few supplementary observations are in some cases sufficient to control the system. The method we propose is intended to focus on this mechanism of order reduction.

Given the generality of the premises, it seems natural to apply the method to a variety of models and observational 
configurations. Its first application to a small size nonlinear model (Lorenz and Emanuel, 1998), together with a mathematical description of the method, can be found in Trevisan and Uboldi (2004), hereafter TU. Results obtained with the proposed assimilation scheme were compared with an experiment in which an Optimal Interpolation type of analysis was used to assimilate the adaptively located observations. Errors, both in the analysis state and in forecasts based on it, were significantly and systematically reduced. A strong increase in the stability of the OAF system was achieved, measured by a strong decrease of the dominant exponent; this is a further indication of the successful control of instabilities. Readers are referred to the paper for further details. A brief description of the method and its implementation can be found in Sect. 2 of the present work. Results obtained with targeted observations in the context of a quasi-geostrophic atmospheric model are described in Sect. 3. Presently, work is being devoted to extending the proposed method to a primitive equation ocean model, and some preliminary results with a standard (i.e. non-targeted) observation network are described in Sect. 4.

\section{Assimilation within the unstable subspace}

In order to confine the assimilation in the unstable subspace, a reliable estimate of the most unstable directions has to be made available at each assimilation time. In principle, the unstable subspace should be formed by the Lyapunov vectors with corresponding positive exponents. In practice, these vectors are estimated by breeding on the data assimilation cycle, as extensively discussed in Carrassi et al. (under review, $2005^{1}$ ), hereafter CTU. The breeding technique introduced by Toth and Kalnay (1997) has been modified to estimate the unstable vectors of the forced OAF system. To estimate the unstable subspace of the OAF system, observations are assimilated in each perturbed trajectory as it is done in the control trajectory. Because the unstable vectors are only approximate estimates of the forecast error, a further step is necessary in order to make them better proxies of the true forecast error structure. At analysis time, a regionalization procedure is used in order to select and isolate, from each bred vector of the current set, one or more "structures", identified as local maxima or minima. This is obtained by means of a Gaussian-shaped modulating function, centered in each maximum or minimum, and applied to the bred vector by point-by-point multiplication. The $N$ vectors obtained in this way have the local structure of one of the current bred vectors and are stored in the $N$ columns of a matrix, $\mathbf{E}$, whose number of rows, $I$, is the state dimension. More details on the regionalization procedure are given, with regard to the

\footnotetext{
${ }^{1}$ Carrassi, A., Trevisan, A., and Uboldi, F.: Deterministic Data Assimilation and Targeting by Breeding on the Data Assimilation System, J. of Atmos. Sci., under review, 2005.
}

specific implementations, in Sects. 3 and 4. The matrix $\mathbf{E}$ is used to estimate the forecast error covariance matrix:

$$
\mathbf{P}^{f}=\mathbf{E} \boldsymbol{\Gamma} \mathbf{E}^{\mathrm{T}}
$$

or, in other words, the analysis increment is confined in the estimated unstable subspace:

$\mathbf{x}^{a}=\mathbf{x}^{f}+\mathbf{E a}$.

Here the $(N, N)$ matrix $\boldsymbol{\Gamma}$ represents the forecast error covariance in the subspace spanned by the columns of $\mathbf{E}$, while the vector of coefficients $\mathbf{a}$ is the control variable, i.e. the analysis increment in that subspace. The mathematical expression of the analysis solution has then the same form as that of Ensemble Kalman Filters (Evensen, 2003), hereafter EnKF, the difference being in how $\mathbf{E}$ is built:

$$
\begin{aligned}
\mathbf{x}^{a}= & \mathbf{x}^{f}+\mathbf{E} \boldsymbol{\Gamma}(\mathbf{H E})^{\mathrm{T}}\left[(\mathbf{H E}) \boldsymbol{\Gamma}(\mathbf{H E})^{\mathrm{T}}+\mathbf{R}\right]^{-1} \\
& \cdot\left(\mathbf{y}^{o}-H\left(\mathbf{x}^{f}\right)\right)
\end{aligned}
$$

or, equivalently, :

$$
\begin{aligned}
\mathbf{x}^{a}= & \mathbf{x}^{f}+\mathbf{E}\left[\boldsymbol{\Gamma}^{-1}+(\mathbf{H E})^{\mathrm{T}} \mathbf{R}^{-1}(\mathbf{H E})\right]^{-1}(\mathbf{H E})^{\mathrm{T}} \\
& \cdot \mathbf{R}^{-1}\left(\mathbf{y}^{o}-H\left(\mathbf{x}^{f}\right)\right)
\end{aligned}
$$

where the vector $\mathbf{y}^{o}$ contains the observations, $\mathbf{R}$ is the observation error covariance matrix and $H$ is the (possibly nonlinear) observation operator, whose Jacobian is $\mathbf{H}$.

In the case of targeted observations, one observation is located in the proximity of the maximum component of each unstable vector, so that the number, $M$, of observations must be at least equal to $N$.

In the case of standard observations, that is to say a fixed observation network, the important question is the following. If we are able to provide a correct estimate of the unstable structures, are these structures located in geographical regions where routine observations are available? If they are, they can be effectively used. Even if, occasionally, the unstable structures go undetected, they might migrate into a more densely observed region, then be detected at a later assimilation time, so that our ability to control the solution is not compromised. In both cases, there are two important points: how large is the number of the unstable structures, and how fast they grow compared to the frequency with which we are able to observe and eliminate them.

The possibility of taking advantage of an existing "standard" fixed observation network is investigated in Sect. 4, in the context of a primitive equations ocean model. It should be pointed out that if the assimilation in the unstable subspace spanned by few bred vectors used at a given analysis time is really efficient, those components of the error are virtually eliminated. If this is the case it becomes necessary to introduce new perturbations. Further comments on this "refreshing" procedure are given in Sects. 3 and 4. 


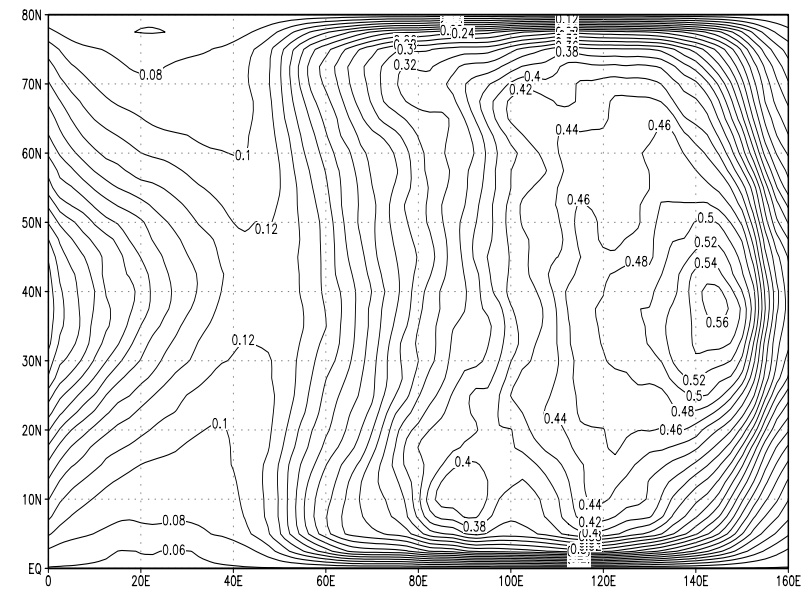

Fig. 1. QG model. Data-dense "land" on longitudes 0-50E. One adaptive observation is located in the data-void "ocean", longitudes 50E-160E. Level and time averaged RMS analysis error, when the adaptive observation is assimilated with 3-D VAR.

\section{Application to a Quasi-Geostrophic atmospheric model}

The method has been applied to an atmospheric quasigeostrophic model (Rotunno and Bao, 1996; Morss, 1999). Results presented in this section are included in CTU, where an extended theoretical discussion, complete results with perfect and noisy observations and a detailed stability analysis can be found. The domain is a periodic channel, with 64 longitude, 33 latitude gridpoints $(16000 \mathrm{~km} \times 8000 \mathrm{~km})$. Potential vorticity is defined on 5 inner vertical levels, and potential temperature is defined at top and bottom boundaries. Observations are assimilated every $12 \mathrm{~h}$. Here we chose perfect model conditions, and perfect observations are assimilated: a long reference model trajectory represents the "true" state evolution, from which observations are taken.

A "land" area is defined, in the western third of the domain (longitudinal grid-points 1-20), where fixed observations are located at each grid-point and assimilated with the 3-D VAR scheme described in Morss (1999). The remaining part of the domain is a wider "ocean" area (longitudes 21-64), on the east in the figures. The fixed observations consist of vertical profiles, measuring temperature and the two components of the horizontal wind. In the wide ocean area, at each analysis time, just one observation is adaptively located (targeted) in the maximum of one unstable vector, bred for 10 days, so that, here, $M=N=1$.

For the reasons discussed at the end of the previous section, the "current" bred vector, that is used in the assimilation, is discarded afterwards, and a new random perturbation starts its breeding cycle. A new bred vector will be ready at the next analysis time $(+12 \mathrm{~h})$, because it has been introduced ( 9 days and $12 \mathrm{~h}$ ) earlier.

Two different experiments, of 2 years simulated time, are performed, with the same trajectory representing the truth.

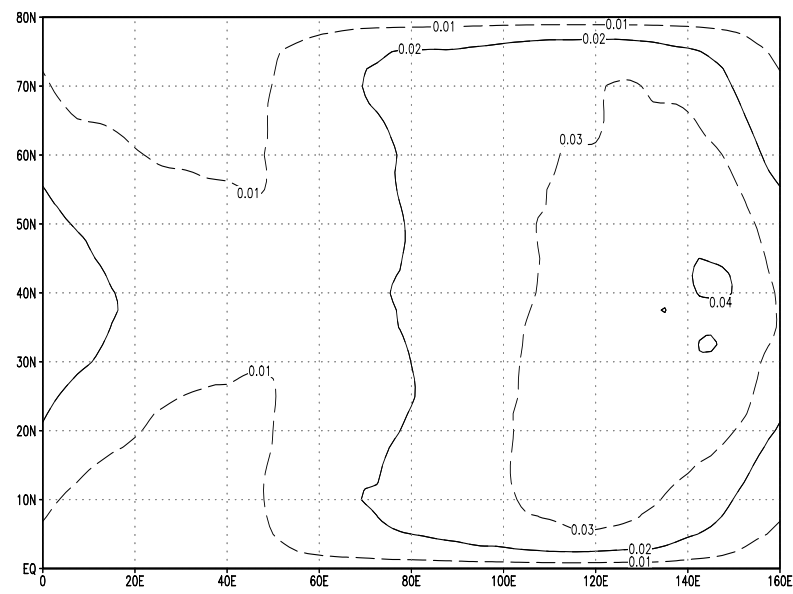

Fig. 2. Same as in Fig. 1, when the adaptive observation is assimilated with the proposed method.

The adaptive observation is assimilated with the 3-D VAR scheme in experiment (1), and with the proposed method in experiment (2), while 3-D VAR is used in both experiments for assimilating the fixed observations over land. Moreover, in experiment (1) the adaptive observation consists of a complete vertical profile (temperature and wind components on 7 levels), while in experiment (2) the adaptive observation consists of a single scalar temperature observation. In both cases the adaptive observation is located in the maximum of the "current" perturbation, so the main difference is in how the adaptive observation is assimilated.

TU noted, in the small model context, the necessity of regionalizing the correction made by using the unstable structure. As explained there, this is basically due to the possible occurrence of regions oppositely correlated, with respect to the main maximum, in the forecast error and in the perturbation used in the assimilation. The forecast error is the superposition of different modes, that can show separate maxima and minima having different amplitude, occasionally opposite sign, with respect to those of the bred vector. So, while the assimilation of the observation effectively eliminates the main error structure, the presence of a secondary one could lead to a correction with the wrong sign. The regionalization is obtained here in the same way as in TU, by means of a modulating function, point-by-point multiplying the "assimilating" perturbation. The choice is a Gaussian function, with $\left(e^{-1}\right)$ decay scale of $2500 \mathrm{~km}$, much larger than the typical scale of single structures.

The maps in Figs. 1 and 2 show the time and height averaged root-mean-square analysis error in the two experiments. The horizontal distribution of the error is quite similar in the two figures, where the maximum of the error is located in the eastern "ocean", affecting the western coast of the "continent". The important feature, anyhow, is the reduction in the error values, which is of about an order of magnitude. 


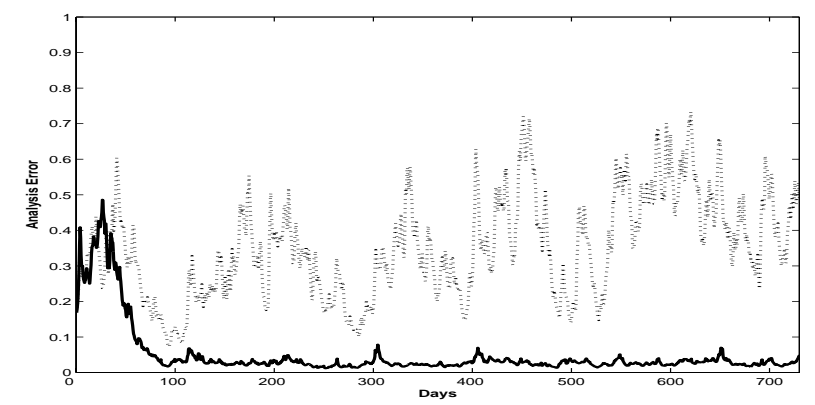

Fig. 3. QG model. RMS analysis error versus time, measured by total enstrophy and normalized by natural variability. 3-D VAR: dotted line. Proposed method: continuous line.

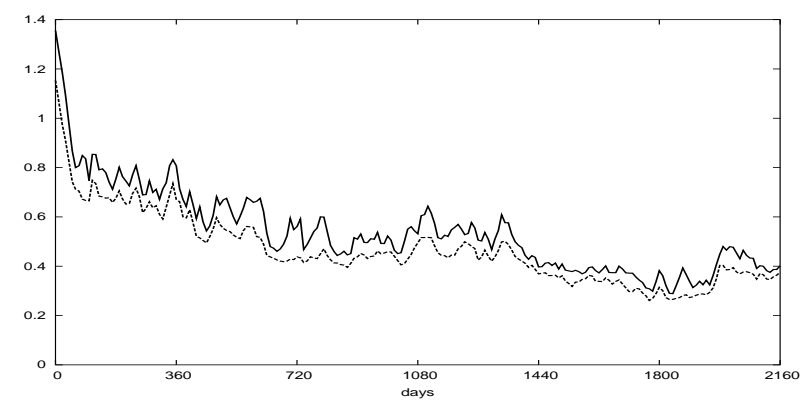

Fig. 4. Ocean model. Forecast (continuous line) and Analysis (dotted line) total energy error (normalized to natural variability) for the standard Cooper-Haines assimilation scheme, starting from a randomly chosen initial "Forecast" state.

Figure 3 shows the analysis error, plotted versus time, for comparison of the results obtained by assimilating the adaptive observation with 3-D VAR (dotted line) or with the proposed method (continuous line).

A detailed stability analysis can be found in CTU. Synthetically, the 3-D VAR experiment shows a positive average growth rate, indicating instability, while with the proposed method a negative growth rate, that is to say stabilization, has been obtained. The value of the average growth rate depends essentially on how the unstable structures develop in the wide ocean area and on how successfully they are controlled by the assimilation of the adaptive observations.

\section{Preliminary results with a primitive equation ocean model}

The method is currently being applied to a primitive equation ocean model, the isopycnal model MICOM (Bleck, 1978; Baraille and Filatoff, 1995), in a simplified configuration: a flat bottom basin, with a $180 \times 140$ horizontal grid and 4 layers, with constant surface wind forcing. The typical dynamic situation is that of a double gyre, cyclonic in the northern half of the domain, anticyclonic in the southern half, with an eastward stream detaching from the western boundary and a

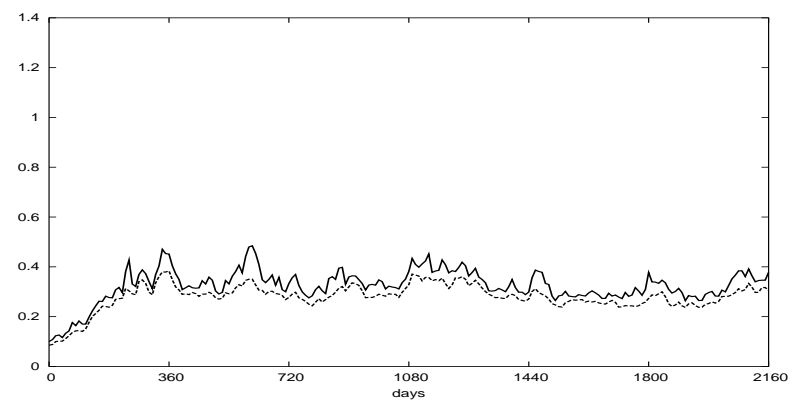

Fig. 5. Same as Fig. 4, but starting from an initial Forecast state whose error has been rescaled to 0.1 of variability.

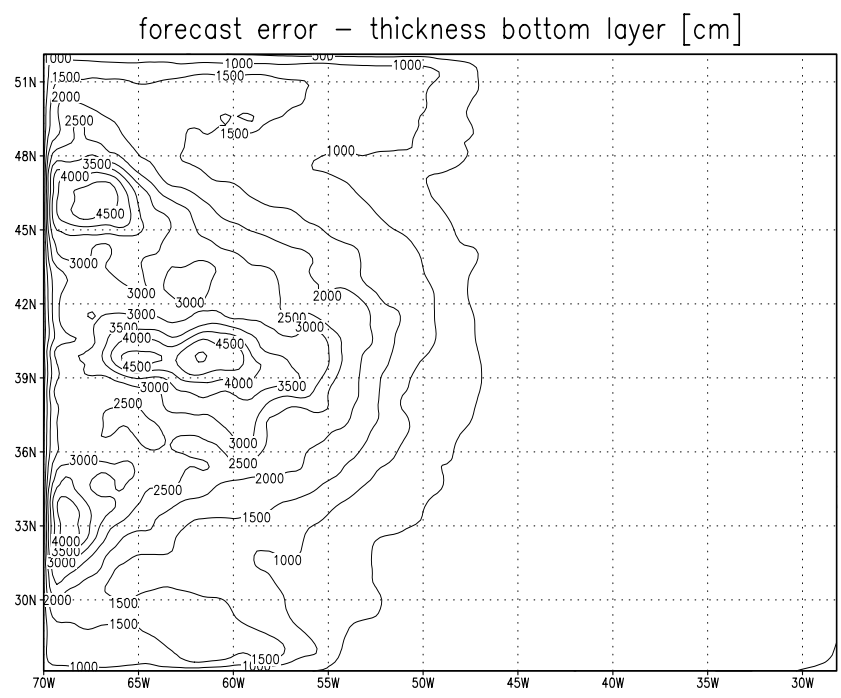

Fig. 6. Ocean model, standard Cooper-Haines analysis. RMS error fields averaged over 6 years: forecast error on thickness of bottom layer.

wide recirculation region in the eastern part of the domain. Again we chose to work with perfect model conditions: a model trajectory represents the "true" trajectory, from which perfect observations are taken. As anticipated in Sect. 2, the case of a fixed "standard" observation network is considered here. Observations consist of sea surface height, available on the whole domain and taken (from the true trajectory) every 10 days. A standard assimilation scheme is also available, the Cooper and Haines (1996) scheme, hereafter CH, based on conservation of linear potential vorticity, and characterized by a homogeneous vertical shift of isopycnals, (internal layers interfaces in the MICOM case), with no change in the bottom pressure, and geostrophic adjustment for velocity. The initial guess of the forecast field is a state chosen randomly from a model trajectory. As can be seen in Fig. 4, the standard $\mathrm{CH}$ assimilation scheme is able to reduce an initially high error to values oscillating between 0.3 and 0.4 of the natural variability. In Fig. 5 the initial error was rescaled to 0.1 of natural variability. The standard $\mathrm{CH}$ is unable to keep the error to its initially low value: the error grows and ends up oscillating again between 0.3 and 0.4 . 


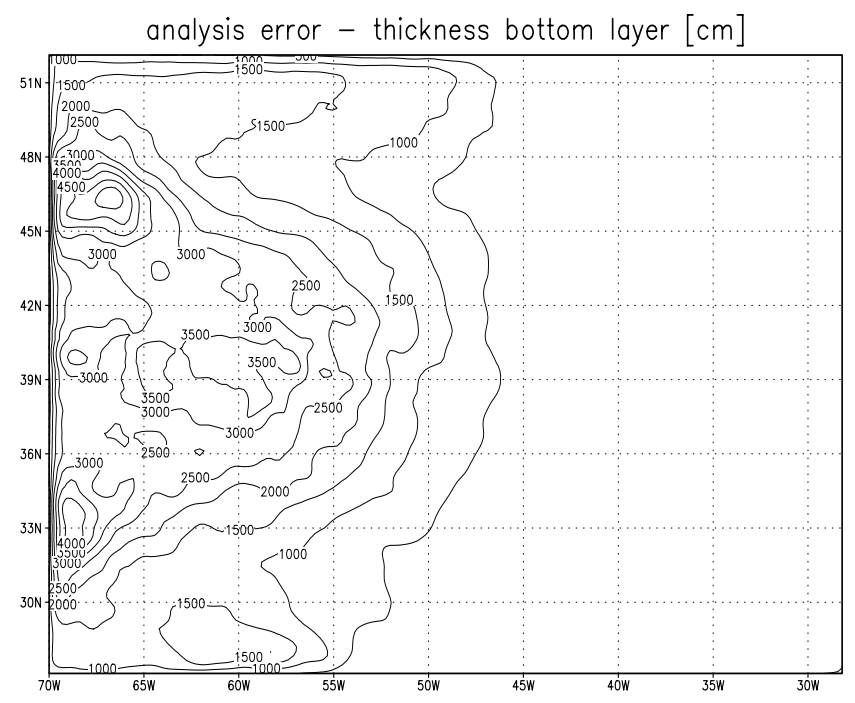

Fig. 7. Same as Fig. 6, analysis error.

Error structures which are well corrected by the $\mathrm{CH}$ scheme are characterized by an error in the vertical position of the density gradient, which can be reduced by modifying the thicknesses of the first and bottom layers only, so that they compensate each other and don't change the bottom pressure. On the other hand, error structures are sometimes compensated internally: there exist surface structures that are not evident in the bottom layer, and bottom structures that are not evident at the surface. Figures 6 and 7 show the 6 years averaged forecast and analysis root-mean-square error on the thickness of the deepest layer 4 (that is to say the elevation from the flat bottom of the interface between layers 3 and 4). It is possible to see that errors present in the forecast are associated with the eastward stream, which usually detaches from the western boundary between latitudes $39 \mathrm{~N}$ and $42 \mathrm{~N}$, while other errors are associated with interactions between the western boundary and the northern cyclonic and southern anticyclonic gyres. The $\mathrm{CH}$ corrections are mostly located in the stream region, but other errors remain, and at least part of them affect the bottom pressure, as it can be seen in Fig. 8 . Moreover, the analysis error is even larger than the forecast error in the northwestern part of the domain. These results support the need for more realistic and flow-dependent vertical covariances in the assimilation of surface height observations.

Work is at present devoted to implement the proposed assimilation method with this ocean model and observation network. The idea is to exploit the information given by the standard observation network described above. As discussed in Sect. 2, ideally the column vectors of the matrix $\mathbf{E}$ are the Lyapunov vectors of the data assimilation system with corresponding positive exponents. To the authors' knowledge there is no practically convenient way to estimate the individual, generally non-orthogonal, Lyapunov vectors (Trevisan and Pancotti, 1998). In the absence of orthogonalization, the bred perturbations are likely to display similar structures. In

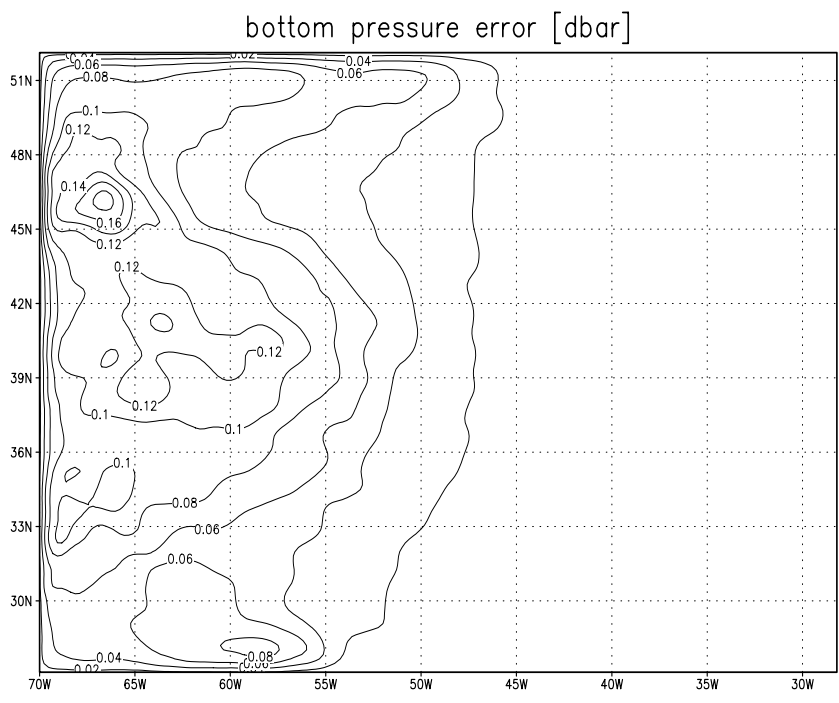

Fig. 8. Same as Figs. 6 and 7, bottom pressure error.

the following we describe the procedure we adopted to identify those structures that are most representative of the unstable vectors and of the forecast error.

A CH analysis scheme starting from a climatological initial forecast state reaches in a year its typical error values, between 0.3 and 0.4 of natural variability. At this point (day 360), perturbations are initially introduced and bred for 2 months, during which the only assimilation running is still $\mathrm{CH}$. This is a first result: a breeding time of about 60 days, which for the ocean is not a long time, is sufficient to produce perturbations whose structures are correlated with structures present in the forecast error. At each assimilation time (every 10 days) a set of 6 independent perturbations are introduced. Initial perturbations are built randomly as differences from model states. At the end of the initial 2 months period (day 420), 36 perturbed states are available. At day 420, the new assimilation system begins to act. Each of the 6 "current" perturbations, the oldest set, is searched for local maxima and minima structures, starting from the largest (in absolute value).

In the current implementation, four steps are taken at analysis time: regionalization, selection, assimilation and refresh.

\section{- Regionalization}

Each of the structures is isolated from the others by means of a modulating function, in a manner analogous to what has been done with the QG model, as described in Sect. 3 (and with the small model in TU). As before, the modulating function is a Gaussian. In this case, anyhow, an elliptical (with elliptical isolines) Gaussian is adapted to each structure, so that 5 coefficients ( 3 for shape and orientation, 2 for center coordinates) are found by resolving a least square problem. A wider Gaussian is then obtained by multiplying its width by a factor 1.5 in the two dimensions. It is necessary to use a wider Gaussian in order to prevent 
an excessive deformation of the structure. Anyhow the Gaussian must not be too wide, because otherwise it may include unwanted secondary structures. By means of this procedure, a (variable) number of "structures" are extracted from the "current" perturbations. A different "localization" technique, which appears systematic, but also computationally expensive (and, perhaps, still risky in areas where unstable structures are only marginally detected by observations) has been proposed by Ott et al. (2004).

\section{- Selection}

A smaller number of these structures is then selected for use in the assimilation, by comparing each of them with the local structure of the innovation (difference between the observations and the forecast estimate) and retaining only those that show a regional correlation larger than 0.8 in absolute value. The correlation is computed by means of a euclidean scalar product between the sea surface elevation component of the structure, He, and the innovation, regionalized with the same Gaussian. The information content of the innovation is rich (in the horizontal directions) in this case. In a more realistic context this comparison could be made with, for example, the portion of satellite tracks which cross the structure. Or, in the case of vertical profiles, by comparing the vertical structure of the innovation with that of the structure. The number of selected structures is usually small, not greater than 8: this is consistent with previous results, obtained with the QG model in Sect. 3 and with the small model in TU. It is also consistent with recent studies which show a local low dimensionality of ensemble structures (Patil et al., 2001; Oczkowski et al., 2005).

The regionalization and selection procedures are performed by considering the surface elevation only, while the modulated perturbations enter the assimilation as full tridimensional fields of state variables.

- Assimilation

The selected structures are stored in the columns of the matrix $\mathbf{E}$ and used in the assimilation. From Eq. 5, by assuming $\mathbf{R}=\sigma_{o}^{2} \mathbf{I}$ and neglecting the resulting term $\sigma_{o}^{2} \boldsymbol{\Gamma}^{-1}$ (perfect observations), the analysis increment is obtained as:

$$
\begin{aligned}
& \mathbf{x}^{a}= \mathbf{x}^{f}+\mathbf{E}\left[(\mathbf{H E})^{\mathrm{T}}(\mathbf{H E})\right]^{-1}(\mathbf{H E})^{\mathrm{T}} \\
& \cdot\left(\mathbf{y}^{o}-H\left(\mathbf{x}^{f}\right)\right)
\end{aligned}
$$

Is is interesting to examine the case of many observations used with a single structure, $M>N=1$. Then the matrix $\mathbf{E}$ has one column only, $\mathbf{E}=\mathbf{e}$, and:

$$
\mathbf{x}^{a}=\mathbf{x}^{f}+\mathbf{e} \frac{(\mathbf{H e})^{\mathrm{T}}\left(\mathbf{y}^{o}-H\left(\mathbf{x}^{f}\right)\right)}{(\mathbf{H e})^{\mathrm{T}}(\mathbf{H e})}
$$

This solution minimizes (in this case $H \mathbf{x}^{a}-$ $H \mathbf{x}^{f}=\mathbf{H}\left(\mathbf{x}^{a}-\mathbf{x}^{f}\right)$ exactly) the expression:

$$
\left(H\left(\mathbf{x}^{a}\right)-\mathbf{y}^{o}\right)^{\mathrm{T}}\left(H\left(\mathbf{x}^{a}\right)-\mathbf{y}^{o}\right)=M I N
$$

subject to the strong constraint (compare with Eq. 3):

$\mathbf{x}^{a}=\mathbf{x}^{f}+\mathbf{e} a$.

Here it can be seen how the analysis increment, constrained by Eq. 9 in the direction of the vector $\mathbf{e}$, has the amplitude that best-fits the observations.

The application of the method is intended here to account for that part of the forecast error belonging to the (estimated) unstable subspace, and that is detected by means of the - supposed unchangeable - standard observation network. A part of the forecast error is however present that is outside the estimated unstable subspace. For this reason the proposed method is used here in combination with the standard analysis. The analysis obtained by means of the described procedure is then used as a background field for a standard $\mathrm{CH}$ analysis. In this case the standard $\mathrm{CH}$ analysis is intended to be augmented rather than replaced by the proposed method.

\section{- Refresh}

Since (perfect) observations are assimilated in the control state and in all the perturbed states, too, the structures which are used in the assimilation are automatically eliminated from all the perturbations (difference fields). This is the mechanism at the base of the problem often referred to, in EnKF literature (Whitaker and Hamill, 2002), as the "rank reduction" of the estimated analysis error covariance matrix. In our method, the effective estimate of the unstable component of the current analysis error could be obtained by using, rather than the "just used" set (from which all "observed" structures were eliminated), the set of vectors that will be used in the next analysis step. If some kind of refreshment is regularly done, this estimate will have a larger dimension, that is to say more unstable structures will be found when the vectors set is searched, than what will be found if no refreshment is done. If, moreover, no refreshment is ever done, all bred vectors (apart from nonlinearities) will tend, as long as the OAF sequence proceeds, to approach each other, and there will be less and less advantage in having sets of more than one bred vector. With the QG model, as described in Sect. 3 (and with the small model in TU), the refreshment is obtained in a drastic way: the "current" bred vector is discarded after the assimilation and replaced by a new random perturbation. Here, in the case of the oceanic primitive equation model and with the standard observation network described above, the current bred vectors, from which the structures have been 


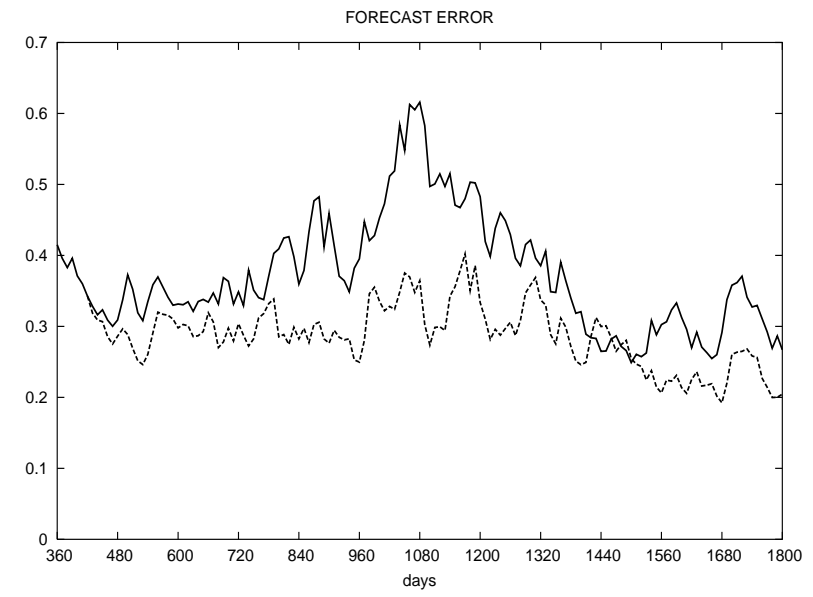

Fig. 9. Ocean model. Total energy forecast error (normalized to natural variability) for the standard $\mathrm{CH}$ assimilation alone (continuous line) and for the proposed "sequential" method (dotted line).

extracted and used in the assimilation, still carry useful information. For example, some other structures are present in deeper layers, besides those evident at the surface at analysis time: such structures may emerge (and hopefully be detected) later on. So, the following procedure has been implemented as a trade-off between keeping or discarding the bred vectors altogether. After the assimilation, one (at turns) of the "current" bred vectors (from which the estimated unstable structures have been extracted) is added to all the other 5 vectors of the same set. One new random perturbation is inserted at its place. The new set of 6 vectors start then to undergo a new 60-day breeding cycle.

The purpose of these experiments is to show how, in the presence of a standard, fixed observation network, our method can be used to eliminate, by means of the assimilation, those unstable structures that are present in the forecast error and that can be detected from the observations available in specific geographical regions. In the present case this means that we can only detect those unstable structures that have an important component in the sea surface elevation. There exist unstable structures, confined in deeper layers, that do not appear in the sea surface elevation field. As long as they don't emerge at the surface, those structure cannot be detected by the fixed observation network used here, but they could be detected by means of adaptive observations, as it is the case for the QG model in Sect. 3. We have performed experiments in which a variable number $N$ of structures are extracted (by regionalization), selected, stored in the $N$ columns of the matrix $\mathbf{E}$ and assimilated by means of Eq. 6 . Results of these experiments (not presented here) show some improvements, that are not, anyhow, systematic. In particular, the sudden growth that appears at about day 980 in the standard assimilation forecast and analysis errors of Figs. 9 and 10 (continuous line), is not satisfactorily controlled. This depends on several factors. One of them is the presence of instabilities in deeper layers that can only be detected by means

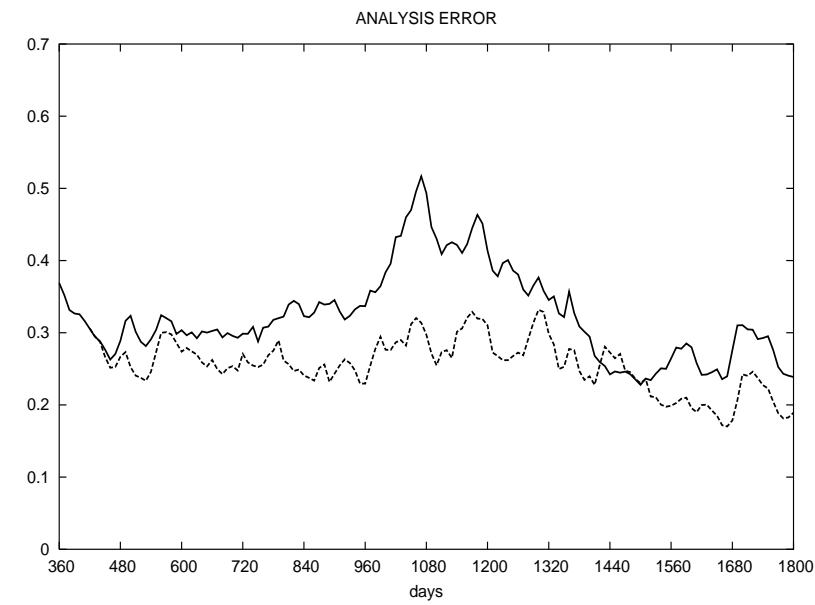

Fig. 10. Same as Fig. 9, analysis error.

of adaptive observations. Another problem that has been diagnosed is the following. The same local structure (or structures with very similar features) appears in two bred vectors of the current set. If the two structures are considered as independent vectors, and both used in the assimilation, the analysis increment can be important in the direction of the difference of the two fields, that, after the regionalization, is not guaranteed to be a well structured unstable direction. This problem was only partially overcome in these experiments, by computing the (surface elevation) correlation between all couples of selected structures, and excluding one of the two if the correlation is larger than 0.99. A simple way of accounting for this problem is obtained by using Eq. 7 with each structure in a sequence, rather than Eq. 6 with all the selected structures together. The sequence concerns the regionalization, selection and assimilation steps, on the control and on all perturbed states. In this way when a structure is eliminated, it is eliminated from all bred vectors and is assimilated only once. The two assimilations differ in regions where structures are overlapping. The results obtained with this sequential procedure are shown (dotted line) in Fig. 9 (forecast error) and Fig. 10 (analysis error), in comparison with the standard $\mathrm{CH}$ assimilation (continuous line). It can be seen that in this way the error peak starting at day 980 is well controlled, as are other secondary peaks. We inspected the characteristics of the flow during the period, lasting approximately six months, when the standard assimilation is unable to control the instabilities and the errors become very large. It appears that, during this period, the meanders of the current are very pronounced and the local structures in the bred vectors are all concentrated and persist longer than usual in the region where the current sharply bends on itself. Such type of a flow appears to be particularly unpredictable and, when this happens, the need to use the dynamically consistent assimilation becomes imperative.

The results obtained show that the method is successful, even with a fixed observation network, in eliminating at least some of the instabilities present in the forecast error, and that, if still present after the analysis, are responsible for 
subsequent error growth. We expect that the use of adaptive observations with this ocean system, by analogy with QG model experiments, will improve the performance of the assimilation.

\section{Conclusions}

The experiments described here are based on the new method proposed in TU, which provides a dynamically consistent analysis state by confining the analysis increment in the unstable subspace of the assimilation system itself. In this way instabilities which are otherwise responsible for error growth are eliminated. With this method it is possible to exploit the observational forcing in the control of instabilities and consequent reduction of the dimension of the assimilation system unstable subspace. Only few unstable structures need to be eliminated at each assimilation time: this can be achieved by means of few adaptively located observations, or exploiting the existing fixed observation networks.

In a quasi-geostrophic atmospheric model, a significant error reduction was obtained compared with a 3-D VAR assimilation, when the estimate of the unstable subspace is used to locate and assimilate adaptive observations

Experiments with a primitive equation ocean model showed that advantage can be taken from a fixed observation network to detect and eliminate at least some of the unstable structures.

Future developments concern the combination of adaptive and standard noisy observations, the assimilation of observations distributed in time, and the approach to the assimilation of real data.

Acknowledgements. We thank R. Morss and M. Corazza for the QG model and its 3-D VAR system, and R. Baraille for the SHOM version of MICOM.

Part of this work has been supported by the projects EPSHOMUBO: 00.87.118.00.470.29.25 and CA2003/03/CMO.

Edited by: S. Vannitsem

Reviewed by: two referees

\section{References}

Baraille, R. and Filatoff, N.: Modèle shallow-water multicouches isopycnal de Miami, Tech. rep., Rapport d'étude SHOM N.003/95, Toulouse, France, 1995.

Bleck, R.: Simulation of Coastal Upwelling Frontogenesis with an Isopycnic Coordinate Model, J. Geophys. Res., 83C, 6163-6172, 1978.

Cooper, M. and Haines, K.: Altimetric Assimilation with Water Property Conservation, J. Geophys. Res., 101C, 1059-1077, 1996.

Evensen, G.: The Ensemble Kalman Filter: Theoretical Formulation and Practical Implementations, Ocean Dynamics, 53, 343367, 2003.

Ghil, M.: Advances in sequential estimation for atmospheric and oceanic flows, J. Meteor. Soc. Japan, 75, 289-304, 1997.

Lorenz, E. N. and Emanuel, K. A.: Optimal Sites for Supplementary Weather Observations: Simulation with a Small Model, J. of Atmos. Sci., 55, 399-414, 1998.

Morss, R.: Adaptive observations: idealized sampling strategies for improving numerical weather prediction., Ph.D. thesis, Massachussets Institute of Technology, Cambridge, MA, USA, 1999.

Oczkowski, M., Szunyogh, I., and Patil, D. J.: Mechanisms for the Development of Locally Low Dimensional Atmospheric Dynamics, J. of Atmos. Sci., in print, 2005.

Ott, E., Hunt, B. H., Szunyogh, I., Zimin, A. V., Kostelich, E. J., Corazza, M., Kalnay, E., Patil, D. J., and Yorke, J. A.: A Local Ensemble Kalman Filter for Atmospheric Data Assimilation, Tellus, 56A, 415-428, 2004.

Patil, D. J., Hunt, B. R., Kalnay, E., Yorke, J. A., and Ott, E.: Local Low Dimensionality of Atmospheric dynamics, Phys. Rev. Lett., 86, 5878-5881, 2001.

Rotunno, R. and Bao, J. W.: A Case Study of Cyclogenesis Using a Model Hyerarchy, Mon. Wea. Rev, 124, 1051-1066, 1996.

Toth, Z. and Kalnay, E.: Ensemble Forecasting at NCEP: the Breeding Method, Mon. Wea. Rev., 125, 3297-3318, 1997.

Trevisan, A. and Pancotti, F.: Periodic Orbits, Lyapunov Vectors, and Singular Vectors in the Lorenz System, J. of Atmos. Sci., 55, 390-398, 1998.

Trevisan, A. and Uboldi, F.: Assimilation of Standard and Targeted Observations in the Unstable Subspace of the ObservationAnalysis-Forecast Cycle System, J. of Atmos. Sci., 61, 103-113, 2004.

Whitaker, J. S. and Hamill, T. M.: Ensemble Data Assimilation without Perturbed Observations, Mon. Wea. Rev., 130, 19131924, 2002. 